\title{
Mdivi-1 and mitochondrial fission: recent insights from fungal pathogens
}

\author{
Barbara Koch ${ }^{1,2} \cdot$ Ana Traven $^{1}$ (1)
}

Received: 30 January 2019 / Revised: 5 February 2019 / Accepted: 13 February 2019 / Published online: 19 February 2019

(c) The Author(s) 2019

\begin{abstract}
Mitochondrial fission shows potential as a therapeutic target in non-infectious human diseases. The compound mdivi- 1 was identified as a mitochondrial fission inhibitor that acts against the evolutionarily conserved mitochondrial fission GTPase Dnm1/Drp1, and shows promising data in pre-clinical models of human pathologies. Two recent studies, however, found no evidence that mdivi-1 acts as a mitochondrial fission inhibitor and proposed other mechanisms. In mammalian cells, Bordt et al. showed that mdivi-1 inhibits complex I in mitochondria (Dev Cell 40:583, 2017). In a second study, we have recently demonstrated that mdivi-1 does not trigger a mitochondrial morphology change in the human yeast pathogen Candida albicans, but impacts on endogenous nitric oxide (NO) levels and inhibits the key virulence property of hyphal formation (Koch et al., Cell Rep 25:2244, 2018). Here we discuss recent insights into mdivi-1's action in pathogenic fungi and the potential and challenges for repurposing it as an anti-infective. We also outline recent findings on the roles of mitochondrial fission in human and plant fungal pathogens, with the goal of starting the conversation on whether the research field of fungal pathogenesis can benefit from efforts in other disease areas aimed at developing therapeutic inhibitors of mitochondrial division.
\end{abstract}

Keywords Mdivi-1 $\cdot$ Mitochondria $\cdot$ Mitochondrial fission $\cdot$ Fungal pathogens $\cdot$ Candida albicans $\cdot$ Cryptococcus neoformans $\cdot$ Aspergillus fumigatus

\section{Introduction}

Millions of people are affected by fungal infections in the world, including a large number of deaths estimated to surpass a million per year (Brown et al. 2012). Fungal cells are fundamentally very similar to mammalian cells. This is generally thought to create a problem for developing antifungal compounds, while minimizing potential toxicity and adverse effects. Fungal models are used to understand human biology and disease conditions (Botstein and Fink 2011; Hartwell 2004; Krobitsch and Lindquist 2000; Menne et al. 2007; Santos et al. 2018; Sun et al. 2016; van Pel et al.

Communicated by M. Kupiec.

Ana Traven

ana.traven@monash.edu

1 Infection and Immunity Program and the Department of Biochemistry and Molecular Biology, Monash University, Clayton, VIC 3800, Australia

2 Present Address: Protein, Science and Engineering, Callaghan Innovation, Christchurch 8140, New Zealand
2013), and the reverse is also possible-in some instances the field of medical mycology might be able to exploit the similarities between fungal and mammalian cells, to build on the knowledge of cellular processes and compounds that are of interest in non-infectious human diseases. One such case is mitochondrial fission, the process by which mitochondria divide.

Mitochondrial fission plays physiological roles in normal cellular functions, promoting distribution of mitochondria between cells during division and elimination of those mitochondria that have been damaged, reviewed in (Friedman and Nunnari 2014; Nunnari and Suomalainen 2012). However, excessive fission, which occurs in response to stressors, during programmed cell death and in human disease pathologies, leads to fragmentation of mitochondria, which in turn causes mitochondrial dysfunction (Ayanga et al. 2016; Cereghetti et al. 2010; Costa et al. 2010; Fannjiang et al. 2004; Frank et al. 2001; Guo et al. 2013; Iqbal and Hood 2014; Lutz et al. 2009; Rambold et al. 2011; Rehman et al. 2012; Song et al. 2011; Wang et al. 2008; Xie et al. 2015). Inhibiting fission of mitochondria could improve mitochondrial and cellular health, and is thought 
of as a potential therapeutic strategy in neurodegenerative and neuropathological conditions, cardiovascular diseases (stroke, heart attack) and cancer (Brooks et al. 2009; Fannjiang et al. 2004; Gomes et al. 2011; Grohm et al. 2012; Ong et al. 2010; Rambold et al. 2011; Rappold et al. 2014; Sharp et al. 2015; Wang et al. 2017; Xie et al. 2015).

Fungal and mammalian machineries for mitochondrial fission are equivalent, and the main factor which performs mitochondrial fission, the GTPase Dnm1 (also known as Drp1), is conserved. Based on this, can the medical mycology field benefit from efforts aimed at developing mitochondrial fission inhibitors (Cassidy-Stone et al. 2008; Lackner and Nunnari 2010; Mallat et al. 2018; Numadate et al. 2014; Qi et al. 2013; Rosdah et al. 2016)? In other words, would mitochondrial fission inhibitors be of use as antifungals? To try to answer this question, here we discuss recent insights into mitochondrial fission in the main human pathogenic fungi (Candida albicans, Cryptococcus neoformans and Aspergillus fumigatus), as well as the important plant pathogen Magnaporthe oryzae (Chang and Doering 2018; Koch et al. 2018; Neubauer et al. 2015; Zhong et al. 2016). We also discuss our recent work on the putative mitochondrial division inhibitor mdivi-1 in C. albicans (Koch et al. 2018). Mdivi-1 was the first specific inhibitor of Dnm1/Drp1 to be discovered (Cassidy-Stone et al. 2008), and follow-on studies indicate therapeutic promise for non-infectious diseases, reviewed in (Rosdah et al. 2016). A complex and somewhat controversial scenario has emerged regarding the mechanism of action of mdivi-1. Two recent studies, ours in C. albicans (Koch et al. 2018) and a previous one in mammalian cells (Bordt et al. 2017) cast doubt on the effect of mdivi-1 on mitochondrial morphology and show other metabolic mechanisms to be involved. Nevertheless, mdivi-1 has activity that is of interest in fungal infections. In this mini-review, we will consider its mechanism of action and potential for repurposing as an antifungal agent.

\section{Mitochondrial fission in pathogenic fungal species}

Our understanding of the fungal mitochondrial fission apparatus is founded in decades of studies in the model yeast Saccharomyces cerevisiae (Fig. 1a) (Labbe et al. 2014). Organelle fission is performed by the Dnm1, which forms rings at the location where mitochondria will divide, and then contracts mitochondria in a process that is coupled to GTP hydrolysis (Bleazard et al. 1999; Ingerman et al. 2005; Mears et al. 2011; Otsuga et al. 1998). Dnm1 is recruited to mitochondria by co-factors: Fis1, a protein in the mitochondrial outer membrane (Mozdy et al. 2000), and Mdv1 that binds to both Fis1 and Dnm1 and brings them together (Cerveny and Jensen 2003; Cerveny et al. 2001; Karren et al. 2005; Tieu and Nunnari 2000; Tieu et al. 2002). Saccharomyces cerevisiae deletion mutants in DNMI, FIS1 or $M D V I$ display a mitochondrial fission defect, resulting in the formation of long, hyper-connected mitochondria, and also have some other cellular phenotypes related to fitness, as well as organelle and membrane structure and contacts (Dimmer et al. 2002; Elbaz-Alon et al. 2014; Gorsich and Shaw 2004; Kanki et al. 2009; Prevost et al. 2018; Qian et al. 2012). Deletion of $C A F 1$, a paralog of $M D V 1$, does not cause a fission defect by itself, but it potentiates the defect of $m d v 1$ mutants (Griffin et al. 2005).

\section{The mitochondrial fission apparatus in pathogenic fungi}

In all four of the pathogenic fungal species that were recently studied (C. albicans, C. neoformans, A. fumigatus and $M$. oryzae), deletion of $D N M 1$ triggered a mitochondrial morphology defect consistent with reduced fission (Chang and Doering 2018; Koch et al. 2018; Neubauer et al. 2015; Zhong et al. 2016) (Fig. 1b). This was expected, given the high conservation of Dnm1 and its roles in mitochondrial fission in eukaryotes. Deletion of the Dnm1 co-factor FISI caused a mitochondrial fission defect in C. neoformans, A. fumigatus and M. oryzae (Chang and Doering 2018; Neubauer et al. 2015; Zhong et al. 2016), consistent with studies in S. cerevisiae (Griffin et al. 2005; Mozdy et al. 2000). However, somewhat surprisingly, we showed that the $C$. albicans fis 1 deletion mutant displays normal mitochondrial morphology (Koch et al. 2018) (Fig. 1B). Regarding Mdv1, homologs could be found in C. neoformans, A. fumigatus and $M$. oryzae and their inactivation triggered a mitochondrial morphology defect (Chang and Doering 2018; Neubauer et al. 2015; Zhong et al. 2016) (Fig. 1b). C. albicans was again different, as its genome does not encode an obvious homolog of Mdv1 (candidagenome.org). Is the mitochondrial fission apparatus in $C$. albicans really all that different to the other fungal species studied? Further work will be needed to address this interesting question. However, we suspect that, rather than a significant departure from the $S$. cerevisiae model, the $C$. albicans Fis 1 homolog does have a role in mitochondrial fission, but perhaps loss of its function can be compensated for by another factor. That other factor could be a divergent, but functional homolog of Mdv1 that can be anchored to the outer mitochondrial membrane to bring Dnm1 to the organelle. Our findings in C. albicans are paralleled by the situation in mammalian cells. In some mammalian cell types, inactivation of Fis1 does not lead to mitochondrial morphology defects, and several other cofactors in the mitochondrial outer membrane can recruit the Dnm1 homolog Drp1 to mitochondria, reviewed in (Labbe et al. 2014). Also, like in C. albicans, no obvious homolog of Mdv1 can be found in mammals (Labbe et al. 2014). 
A

Dnml $\widetilde{F}$ Fisl $\approx \mathrm{Mdv1}$

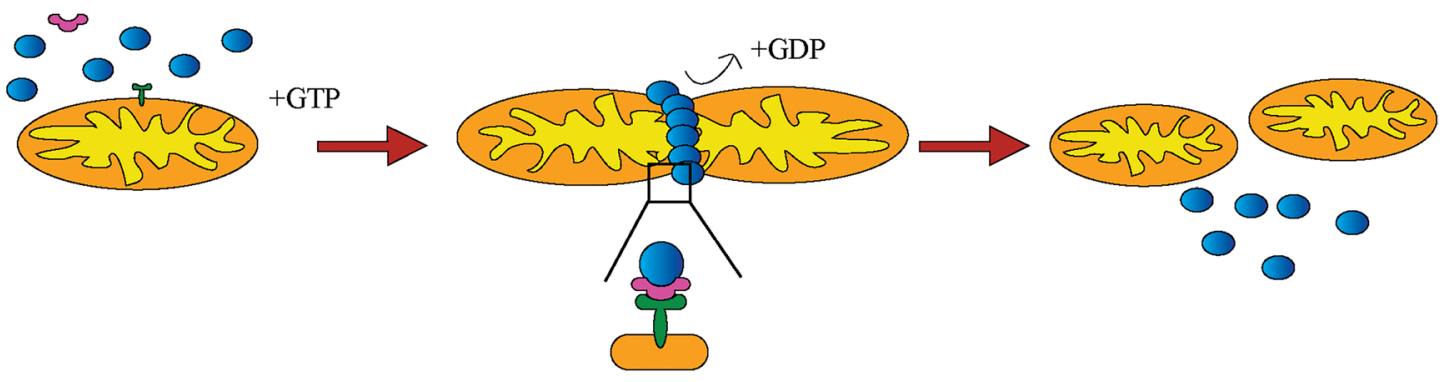

B

S. cerevisiae
dnml
fisl
$m d v 1$

C. albicans

dnm I

fis 1

$m d v 1$

A. fumigatus

dnm 1

fisl

$m d v 1$

M. oryzae

dinm I
Defective mitochondrial Defective growth
morphology

$\mathrm{NO}$

NO

NO

$\mathrm{NO}$

NO

not conserved

YES

YES

YES

YES

YES

YES

YES
Fig. 1 The mitochondrial fission apparatus in fungal species. a Cartoon of the mitochondrial fission machinery as known in S. cerevisiae. b Effects on mitochondrial morphology and growth following inactivation of mitochondrial fission components in fungal species. For those species where we indicate no growth defect, this is under standard and optimal laboratory conditions. Some cellular fitness

\section{Impact of mitochondrial fission of cellular growth and fitness of fungal pathogens}

Disrupting mitochondrial fission had drastically different effect on growth rates and cellular fitness between yeasts and filamentous fungi. In the yeasts $C$. albicans and $C$. neoformans mitochondrial fission mutants did not show any obvious growth defects in vitro, not even under stressful conditions such as elevated temperature, non-preferred and non-fermentable carbon sources or in response to stressors (Chang and Doering 2018; Koch et al. 2018) and changes have been reported for the $S$. cerevisiae mutants under specific conditions, but we want to make the point that the general fitness effects of mitochondrial fission mutations are profoundly different between yeast and filamentous fungi. Data for pathogens are based on Chang and Doering (2018), Koch et al. (2018), Neubauer et al. (2015) and Zhong et al. (2016)

(Koch and Traven unpublished). The C. neoformans mitochondrial fission mutants also displayed normal growth in vivo in the lung in the murine inhalation model of cryptococcal infections, and survived normally in innate immune phagocytes (macrophages) in vitro (Chang and Doering 2018). In contrast, in the filamentous pathogens A. fumigatus and $M$. oryzae mitochondrial fission mutants showed drastically reduced hyphal growth in vitro (Neubauer et al. 2015; Zhong et al. 2016), and the M. oryzae mitochondrial fission mutants were less virulent in a plant infection model (Zhong et al. 2016). Virulence of the $A$. 
fumigatus mitochondrial fission mutants was only tested in the Galleria mellonella larvae model, where they displayed normal virulence (Neubauer et al. 2015). It remains to be seen how they grow and infect a mammalian host. The conclusion that inactivation of mitochondrial fission has a bigger effect on cellular growth in filamentous fungi compared to yeasts is supported by the previous work in model fungal species (Gerstenberger et al. 2012; Mozdy et al. 2000; Otsuga et al. 1998).

\section{Is mitochondrial fission important for filamentous hyphal growth of yeast species?}

Since mitochondrial fission is important for hyphal growth of the filamentous molds A. fumigatus and M. oryzae, an interesting question is whether it is necessary for hyphal growth of a yeast species. Our data in $C$. albicans suggest "no". While C. albicans grows in yeast morphology under default conditions, it forms filamentous hyphae in response to a variety of environmental signals. This process of morphology change is linked to pathogenicity (Sudbery 2011). The C. albicans dnm 1 displayed a modest shortening of invasive hyphae on solid medium (plates) (Koch et al. 2018). However, in liquid medium, where there is no resistance to hyphal growth, it formed wild type-looking hyphae (Koch et al. 2018). The shorter hyphae on solid medium suggested to us that perhaps the $d n m 1$ mutant strain might form hyphae with less invasive capacity. We tested this idea using the worm infection model, because worm killing by $C$. albicans depends on the ability of fungal hyphae to penetrate the animal's cuticle. Indeed, the $d n m l$ mutant was moderately less virulent in the worm model (Fig. 2). Whether reduced hyphal invasion potential of the C. albicans dnml mutant translates to reduced virulence in mice awaits testing.

\section{So, is inhibiting mitochondrial fission promising as an antifungal strategy?}

Based on the growth defects of mitochondrial fission mutants, it could be for filamentous pathogens. It is, however, worth noting that recent work showed that killing of A. fumigatus by human immune cells results in mitochondrial fragmentation (Ruf et al. 2018). This would suggest that inhibiting mitochondrial fission might help the pathogen to evade immune responses. Hyper-fused mitochondria have also been previously linked to increase survival of Cryptococcus gattii in macrophages (Ma et al. 2009; Voelz et al. 2014). Clearly, much more fundamental knowledge is needed on the roles of mitochondrial fission in fungal pathogenesis before we know if its targeting is warranted for therapy.

\section{The putative mitochondrial fission inhibitor mdivi-1: what roles does it play in fungal pathogens?}

Ten years ago, the lab of Jodi Nunnari reported the discovery of mdivi-1, a compound that triggers a mitochondrial fission defect (Cassidy-Stone et al. 2008). The mechanism is conserved between the model yeast $S$. cerevisiae and mammalian cells, and it involves allosteric inhibition of Dnm1 self-assembly into rings. That Dnm1 is the mdivi-1 target was established with in vitro experiments with the purified yeast protein (assaying for GTPase activity and the effects of mdivi-1 on the formation of Dnm1 spirals using electron microscopy), and also by target overexpression with mammalian Drp1 in cell culture to demonstrate reduced effects of the compound (Cassidy-Stone et al. 2008). A subsequent study used in vitro GTPase activity assays to show that mdivi-1 inhibits mammalian Drp1 (Numadate et al. 2014), and a recent review of the literature concluded that, overall, there is substantial experimental support for the notion that mdivi- 1 inhibits mitochondrial fission in mammalian cells (Smith and Gallo 2017). Mdivi-1 has shown therapeutic promise in a range of disease models, including animal studies of cardiovascular dysfunction, brain cancer, and Parkinson's disease (Brooks et al. 2009; Grohm et al. 2012; Lackner and Nunnari 2010; Ong et al. 2010; Rappold et al. 2014; Rehman et al. 2012; Wang et al. 2017; Xie et al. 2015).

\section{Mdivi-1 might not always inhibit mitochondrial fission}

The notion that the primary cellular target of mdivi-1 is Dnm1 and mitochondrial fission was challenged by two recent reports: (1) we showed that mdivi-1 does not cause a steady state mitochondrial morphology defect in the pathogenic yeast $C$. albicans, and deletion of DNMI does not replicate the phenotypic effects of mdivi-1 on C. albicans cells (Koch et al. 2018). Our results contrast with the reported inhibition of mitochondrial fission by mdivi-1 in S. cerevisiae (Cassidy-Stone et al. 2008). (2) Bordt et al. showed that mdivi-1 does not trigger changes in mitochondrial morphology in mammalian cells and it does not inhibit mammalian Drp1 GTPase activity in vitro (Bordt et al. 2017). Their results contrast with the original study by Cassidy-Stone et al. (2008) and subsequent reports in various mammalian systems, including the aforementioned demonstration of mdivi-1-dependent inhibition of Drp1 in vitro (Numadate et al. 2014). Bordt et al. were, however, able to show that mdivi-1 inhibits $S$. cerevisiae Dnm1 in vitro (Bordt et al. 2017), similarly to the initial report 

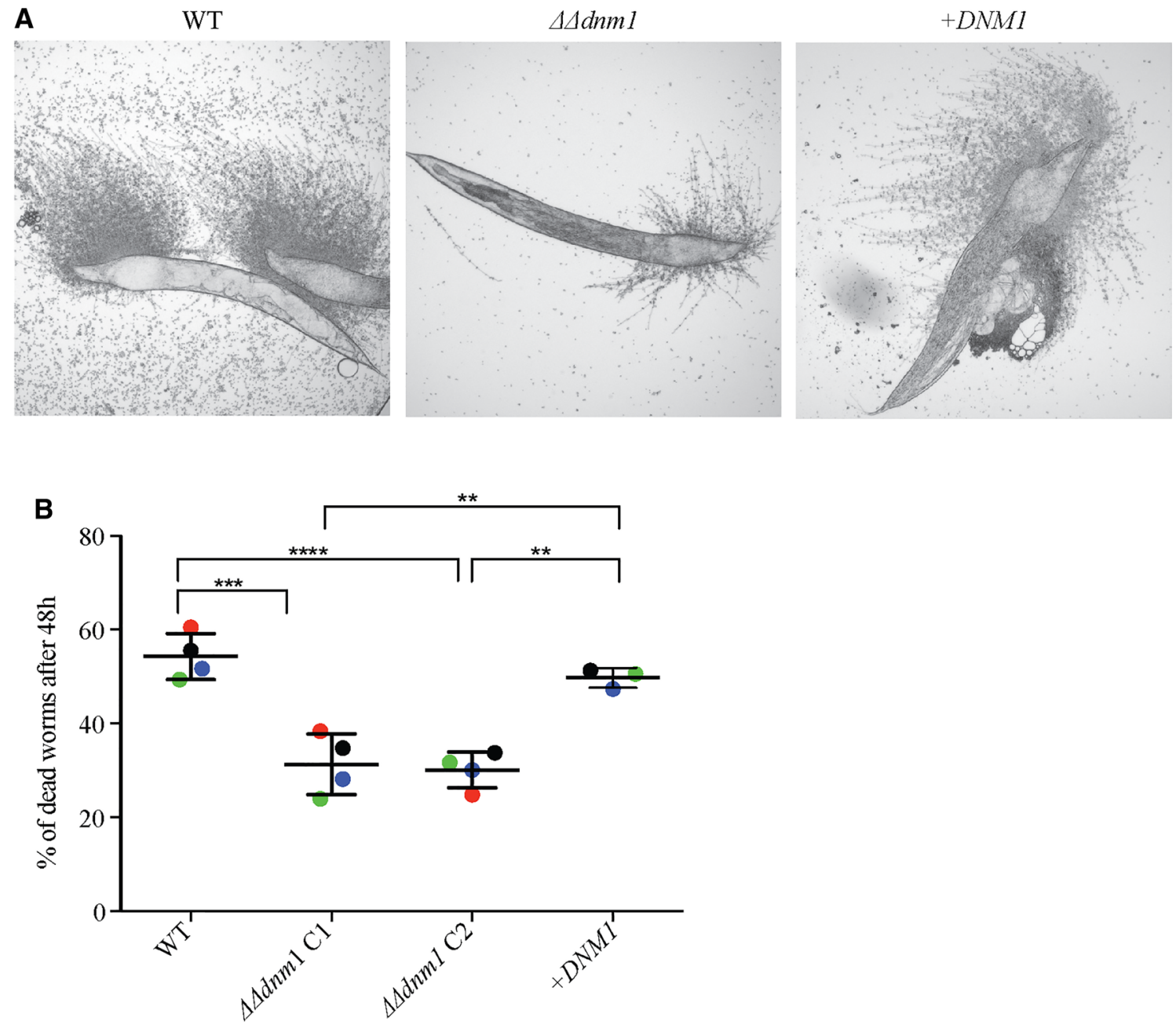

Fig. 2 C. albicans Dnm1 plays a role in hyphal invasion in the worm infection model. a The worm C. elegans ( $g l p-4$; sek-1) was infected with $C$. albicans strains as follows: wild type, a $D N M 1$ deletion strain $\Delta \Delta d n m 1$ or a complemented mutant strain $(+D N M 1)$. The strains are described in (Koch et al. 2018), and the worm C. albicans infection method is described in detail in our previous publication (Koch et al. 2017). Shown are representative images after $48 \mathrm{~h}$ of infection. b The worm infection protocol was performed as in a and dead worms killed due to penetrative hyphal growth of C. albicans were counted

by Cassidy-Stone et al. (2008), opening up the possibility that mdivi-1 has different effects on fungal and mammalian Dnm1. But is $S$. cerevisiae a good model for other fungal species in this case? As mentioned, our study in $C$. albicans showed the opposite results regarding the ability of mdivi-1 to inhibition of mitochondrial fission to what has been shown in S. cerevisiae ("yes" in S. cerevisiae and "no" in C. albicans) (Koch et al. 2018), (Cassidy-Stone et al. 2008). We are aware of only one other fungal species in which the effects of mdivi-1 on mitochondrial morphology were tested. In the filamentous pathogen $M$. oryzae mdivi-1 did trigger a mitochondrial morphology change towards less punctate and more fused structures, consistent after $48 \mathrm{~h}$. Two independent $\Delta \Delta d n m 1$ strains were used (labeled as $\mathrm{C} 1$ and $\mathrm{C} 2$ ). Four biological replicates were done for the wild type and the $\Delta \Delta d n m 1$ strain, while the complemented $(+D N M 1)$ strain was assayed in three of the biological replicates. The independent experiments are color coded. Shown are the mean and standard deviation. Statistical significance was determined with one-way ANOVA and Tukey's multiple comparisons test. ${ }^{*} p>0.001, * * * p>0.0001$, $* * * * p<0.0001$

with inhibition of organellar fission (Zhong et al. 2016). Why these contrasting effects of mdivi-1 are seen in different systems is not clear at the moment. "Trivial" explanations such as the source of the compound, concentrations used, stock solutions, and solubility in water (mdivi-1 is poorly water-soluble), do not appear to be the reason for the discrepancies in mammalian systems (Smith and Gallo 2017). Regarding fungi, we do not know why $C$. albicans behaves differently to $S$. cerevisiae and $M$. oryzae, but we note the differences in the mitochondrial fission apparatus in $C$. albicans compared to the other fungi, as shown in our recent study (Koch et al. 2018), and discussed above. 
Mdivi-1 is a novel inhibitor of hyphal growth in $C$. albicans

Although it did not affect mitochondrial morphology in C. albicans, mdivi-1 inhibited hyphal growth (Koch et al. 2018). Since hyphal formation is an important virulencerelated process, we decided to discern the processes affected by mdivi-1 in $C$. albicans. A detailed RNAseq experiments over a time course of $2 \mathrm{~h}$ following addition of mdivi-1 to a hyphal culture revealed that transcript levels for genes expressed during hyphal growth were reduced, and there were several changes to metabolism-related gene expression indicative of mitochondrial and metabolic stress (Koch et al. 2018). Genes encoding subunits of the mitochondrial respiratory complexes were transiently inhibited by mdivi- 1 and, at the same time expression of alternative oxidases was increased. These results suggested that perhaps mdivi-1 inhibited mitochondrial respiration in some way, which would be consistent with the study of Bordt et al. that reported that the second target of mdivi-1 in mammalian cells is respiratory complex I (Bordt et al. 2017). While we cannot exclude this mechanism, treatment of $C$. albicans with mdivi-1 did not phenocopy complex I mutants with respect to growth phenotypes (Koch et al. 2018). Other transcriptional changes related to metabolism include upregulation of the glyoxylate cycle, gluconeogenesis and fatty acid oxidation, as well as a large upregulation of amino acid biosynthesis-related genes, particularly arginine biosynthesis. Some of the these metabolic changes triggered by mdivi-1 also occur to C. albicans following phagocytosis by macrophages (Lorenz et al. 2004; Tucey et al. 2018), indicating that mdivi-1 creates a metabolic situation which is in part similar to the macrophage phagosome. However, C. albicans transitions from yeast to hyphae in macrophages, while in the presence of mdivi-1 it cannot transition from yeast to hyphae, and it also cannot maintain hyphal growth when mdivi-1 is added to pre-formed hyphae (Koch et al. 2018). How metabolic stress signals control hyphal formation in these different scenarios remains to be understood.

\section{Another mdivi-1-dependent cellular pathway discovered in C. albicans}

In addition to metabolic changes, we showed that mdivi-1 triggered a reduction of endogenous nitric oxide (NO) levels in $C$. albicans cells, and used this discovery to show for the first time that endogenous NO plays an important role in hyphal formation by $C$. albicans (Koch et al. 2018). Our data suggest that the main transcriptional repressor of hyphae-specific genes, $\mathrm{Nrg} 1$, is downstream of mdivi-1 and NO-dependent regulation of hyphal gene expression and hyphal growth (Koch et al. 2018). With these data, we established that, in addition to mitochondrial morphology and respiration via complex I inhibition, mdivi-1 also targets NO-dependent signaling in cells. Signaling via NO is an important physiological process in mammalian cells, and it will be interesting to test if mdivi-1 interferes with it in mammalian systems.

\section{Mdivi-1 as an antifungal agent?}

Development of mdivi- 1 as human therapeutic agent is of interest, but, as recently reviewed, it is not trivial (Rosdah et al. 2016). Mdivi-1 is poorly soluble in water, and in our study in $C$. albicans we used a fungal strain in which the genes encoding major efflux pumps were deleted (Koch et al. 2018). Comparing this strain with an efflux-competent strain is indicative of mdivi-1 being effluxed. Also, the safety and pharmacological properties of mdivi-1 are still to be characterised in detail (Rosdah et al. 2016).

\section{Effects of mdivi-1 on fungi in infection}

Treatment with mdivi-1 reduced infection of barley leaves with M. oryzae (Zhong et al. 2016), showing promising antifungal properties in a plant infection model. Regarding human fungal pathogens, our study in C. albicans is to our knowledge the first one to test the effects of mdivi-1 on virulence-related biology. When mdivi-1 was added to C. albicans macrophage co-cultures, hyphal formation was represssed and two important hyphae-dependent immune cell processes were compromised: cell death of macrophages caused by $C$. albicans infection was reduced, and macrophages were not inducing maturation of the inflammatory cytokine IL- $1 \beta$ as efficiently as in control conditions (Koch et al. 2018). Mdivi-1 also reduced hyphae that $C$. albicans makes in the worm C. elegans (Koch et al. 2018). How mdivi-1 behaves in a mammalian model of fungal infection has not been tested yet. The effects of mdivi-1 in vitro suggest that this compound could modulate levels of inflammation and reduce hyphae-dependent pathogenicity.

\section{Effects of mdivi-1 on immune cells in infection}

If it was to be used as antifungal agent, mdivi-1 would not only target fungal cells, but would also have an effect on host cells. A recent manuscript reported that mdivi-1, when coupled with a mitochondrial fusion-promoting compound M1, is able to drive the formation of more fused mitochondrial network structure in T cells, which had beneficial effects in mouse models of tumorigenesis and infection (Buck et al. 2016). When T cells that were treated ex vivo with mdivi-1 plus M1 were transferred into mice, the animals mounted an improved $\mathrm{T}$ cell response to the bacterium Listeria monocytogenes (Buck et al. 2016). The same study also 
showed that mdivi-1 reduced the switch to Warburg metabolism (aerobic glycolysis) in murine bone marrow-derived macrophages, which were activated by the bacterial ligand lipopolysaccharide (LPS) and interferon gamma. In the same scenario, mdivi-1 also reduced the expression of Nos2 in macrophages, which is the nitric oxide synthase needed for making NO to kill microbial invaders. A similar effect to reduce aerobic glycolysis was seen in LPS-treated dendritic cells. It is important to note that in both macrophages and dendritic cells, the switch to increased aerobic glycolysis upon encountering LPS was still occurring in a substantial manner in mdivi-1-treated cells, and mdivi-1-treated macrophages expressed Nos 2 upon LPS activation above resting levels (Buck et al. 2016). This suggests that mdivi-1 reduces, but does not prevent innate immune activation by LPS.

The switch of innate immune cells to aerobic glycolysis upon activation is a conserved feature and also occurs upon C. albicans challenge, where it is important for mounting cytokine responses and survival of mice upon systemic infection (Cheng et al. 2016; Dominguez-Andres et al. 2017). However, we have recently shown that the absolute reliance of macrophages on glycolysis once they switch to Warburg metabolism opens a window of opportunity for C. albicans, whereby the pathogen can outcompete macrophages for glucose and kill them in the process (Tucey et al. 2018). Therefore, modulation of glucose homeostasis and immune cell metabolism might require a balance for optimal antimicrobial responses, which mdivi-1 might help to achieve. Reduction of the glycolytic shift in activated immune cells by mdivi-1 might also act to dampen inflammation and prevent hyper-inflammatory pathology in infection. Since mdivi-1 has mostly been studied as a drug lead in non-infectious condition, its potential benefits in infection are highly speculative at the moment. Further work should clarify its effects on fungal pathogens and immune cells in infection.

Acknowledgements Work in the Traven lab on mitochondrial morphology and mdivi-1 in C. albicans was supported by a project grant from the Australian National Health and Medical Research Council (NHMRC) (APP1101562). Barbara Koch was funded by a postdoctoral fellowship from the Austrian Science Fund.

OpenAccess This article is distributed under the terms of the Creative Commons Attribution 4.0 International License (http://creativeco mmons.org/licenses/by/4.0/), which permits unrestricted use, distribution, and reproduction in any medium, provided you give appropriate credit to the original author(s) and the source, provide a link to the Creative Commons license, and indicate if changes were made.

\section{References}

Ayanga BA, Badal SS, Wang Y, Galvan DL, Chang BH, Schumacker PT, Danesh FR (2016) Dynamin-related protein 1 deficiency improves mitochondrial fitness and protects against progression of diabetic nephropathy. JASN 27:2733-2747. https://doi. org/10.1681/asn.2015101096

Bleazard W et al (1999) The dynamin-related GTPase Dnm1 regulates mitochondrial fission in yeast. Nat Cell Biol 1:298-304. https:// doi.org/10.1038/13014

Bordt EA et al (2017) The putative Drp1 inhibitor mdivi-1 is a reversible mitochondrial complex I inhibitor that modulates reactive oxygen. Species Dev Cell 40:583-594.e586. https:// doi.org/10.1016/j.devcel.2017.02.020

Botstein D, Fink GR (2011) Yeast: an experimental organism for 21st century biology. Genetics 189:695-704. https://doi.org/10.1534/ genetics.111.130765

Brooks C, Wei Q, Cho SG, Dong Z (2009) Regulation of mitochondrial dynamics in acute kidney injury in cell culture and rodent models. J Clin Investig 119:1275-1285. https://doi.org/10.1172/ jci37829

Brown GD, Denning DW, Gow NA, Levitz SM, Netea MG, White TC (2012) Hidden killers: human fungal infections. Sci Transl Med 4:165rv113. https://doi.org/10.1126/scitranslmed.3004404

Buck MD et al (2016) Mitochondrial dynamics controls T cell fate through. Metab Progr Cell 166:63-76. https://doi.org/10.1016/j. cell.2016.05.035

Cassidy-Stone A et al (2008) Chemical inhibition of the mitochondrial division dynamin reveals its role in Bax/Bak-dependent mitochondrial outer membrane permeabilization. Dev Cell 14:193-204. https://doi.org/10.1016/j.devcel.2007.11.019

Cereghetti GM, Costa V, Scorrano L (2010) Inhibition of Drp1-dependent mitochondrial fragmentation and apoptosis by a polypeptide antagonist of calcineurin. Cell Death Differ 17:1785. https://doi. org/10.1038/cdd.2010.61

Cerveny KL, Jensen RE (2003) The WD-repeats of Net2p interact with Dnm1p and Fis $1 p$ to regulate division of mitochondria. Mol Biol Cell 14:4126-4139. https://doi.org/10.1091/mbc.e03-02-0092

Cerveny KL, McCaffery JM, Jensen RE (2001) Division of mitochondria requires a novel DNM1-interacting protein, Net2p. Mol Biol Cell 12:309-321. https://doi.org/10.1091/mbc.12.2.309

Chang AL, Doering TL (2018) Maintenance of mitochondrial morphology in Cryptococcus neoformans is critical for stress resistance and virulence. mBio. https://doi.org/10.1128/mBio.01375-18

Cheng SC et al (2016) Broad defects in the energy metabolism of leukocytes underlie immunoparalysis in sepsis. Nat Immunol 17:406-413. https://doi.org/10.1038/ni.3398

Costa V et al (2010) Mitochondrial fission and cristae disruption increase the response of cell models of Huntington's disease to apoptotic stimuli. EMBO Mol Med 2:490-503. https://doi. org/10.1002/emmm.201000102

Dimmer KS, Fritz S, Fuchs F, Messerschmitt M, Weinbach N, Neupert W, Westermann B (2002) Genetic basis of mitochondrial function and morphology in Saccharomyces cerevisiae. Mol Biol Cell 13:847-853. https://doi.org/10.1091/mbc.01-12-0588

Dominguez-Andres J et al (2017) Rewiring monocyte glucose metabolism via C-type lectin signaling protects against disseminated candidiasis. PLoS Pathog 13:e1006632. https://doi.org/10.1371/ journal.ppat.1006632

Elbaz-Alon Y, Rosenfeld-Gur E, Shinder V, Futerman AH, Geiger $\mathrm{T}$, Schuldiner M (2014) A dynamic interface between vacuoles and mitochondria in yeast. Dev Cell 30:95-102. https://doi. org/10.1016/j.devcel.2014.06.007

Fannjiang Y et al (2004) Mitochondrial fission proteins regulate programmed cell death in yeast. Genes Dev 18:2785-2797. https:// doi.org/10.1101/gad.1247904

Frank S et al (2001) The role of dynamin-related protein 1, a mediator of mitochondrial fission in apoptosis. Dev Cell 1:515-525

Friedman JR, Nunnari J (2014) Mitochondrial form and function. Nature 505:335-343. https://doi.org/10.1038/nature12985 
Gerstenberger JP, Occhipinti P, Gladfelter AS (2012) Heterogeneity in mitochondrial morphology and membrane potential is independent of the nuclear division cycle in multinucleate fungal cells. Eukaryot Cell 11:353-367 https://doi.org/10.1128/ec.05257-11

Gomes LC, Di Benedetto G, Scorrano L (2011) During autophagy mitochondria elongate, are spared from degradation and sustain cell viability. Nat Cell Biol 13:589-598. https://doi.org/10.1038/ ncb2220

Gorsich SW, Shaw JM (2004) Importance of mitochondrial dynamics during meiosis and sporulation. Mol Biol Cell 15:4369-4381. https://doi.org/10.1091/mbc.e03-12-0875

Griffin EE, Graumann J, Chan DC (2005) The WD40 protein Caf4p is a component of the mitochondrial fission machinery and recruits Dnm1p to mitochondria. J Cell Biol 170:237-248. https://doi. org/10.1083/jcb.200503148

Grohm J et al (2012) Inhibition of Drp1 provides neuroprotection in vitro and in vivo. Cell Death Differ 19:1446-1458. https://doi. org/10.1038/cdd.2012.18

Guo X, Disatnik MH, Monbureau M, Shamloo M, Mochly-Rosen D, Qi X (2013) Inhibition of mitochondrial fragmentation diminishes Huntington's disease-associated neurodegeneration. J Clin Investig 123:5371-5388. https://doi.org/10.1172/jci70911

Hartwell LH (2004) Yeast and cancer. Biosci Rep 24:523-544 https:// doi.org/10.1007/s10540-005-2743-6

Ingerman E, Perkins EM, Marino M, Mears JA, McCaffery JM, Hinshaw JE, Nunnari J (2005) Dnm1 forms spirals that are structurally tailored to fit mitochondria. J Cell Biol 170:1021-1027. https ://doi.org/10.1083/jcb.200506078

Iqbal S, Hood DA (2014) Oxidative stress-induced mitochondrial fragmentation and movement in skeletal muscle myoblasts. Am J Physiol Cell Physiol 306:C1176-C1183. https://doi.org/10.1152/ ajpcell.00017.2014

Kanki T et al (2009) A genomic screen for yeast mutants defective in selective mitochondria autophagy. Mol Biol Cell 20:4730-4738. https://doi.org/10.1091/mbc.E09-03-0225

Karren MA, Coonrod EM, Anderson TK, Shaw JM (2005) The role of Fis $1 \mathrm{p}-\mathrm{Mdv} 1 \mathrm{p}$ interactions in mitochondrial fission complex assembly. J Cell Biol 171:291-301. https://doi.org/10.1083/ jcb. 200506158

Koch B, Tucey TM, Lo TL, Novakovic S, Boag P, Traven A (2017) The mitochondrial GTPase Gem 1 contributes to the cell wall stress response and invasive growth of Candida albicans. Front Microbiol 8:2555. https://doi.org/10.3389/fmicb.2017.02555

Koch B et al (2018) A metabolic checkpoint for the yeast-to-hyphae developmental switch regulated by endogenous nitric oxide. Signal Cell Rep 25:2244-2258.e2247. https://doi.org/10.1016/j.celre p.2018.10.080

Krobitsch S, Lindquist S (2000) Aggregation of huntingtin in yeast varies with the length of the polyglutamine expansion and the expression of chaperone proteins. Proc Natl Acad Sci USA 97:1589-1594

Labbe K, Murley A, Nunnari J (2014) Determinants and functions of mitochondrial behavior. Annu Rev Cell Dev Biol 30:357-391. https://doi.org/10.1146/annurev-cellbio-101011-155756

Lackner LL, Nunnari J (2010) Small molecule inhibitors of mitochondrial division: tools that translate basic biological research into medicine. Chem Biol 17:578-583. https://doi.org/10.1016/j. chembiol.2010.05.016

Lorenz MC, Bender JA, Fink GR (2004) Transcriptional response of Candida albicans upon internalization by macrophages. Eukaryot Cell 3:1076-1087. https://doi.org/10.1128/ec.3.5.1076-1087.2004

Lutz AK et al (2009) Loss of parkin or PINK1 function increases Drp1dependent mitochondrial fragmentation. J Biol Chem 284:2293822951. https://doi.org/10.1074/jbc.M109.035774

$\mathrm{Ma} \mathrm{H}$ et al (2009) The fatal fungal outbreak on Vancouver Island is characterized by enhanced intracellular parasitism driven by mitochondrial regulation. Proc Natl Acad Sci USA 106:12980 12985. https://doi.org/10.1073/pnas.0902963106

Mallat A et al (2018) Discovery and characterization of selective small molecule inhibitors of the mammalian mitochondrial division dynamin, DRP1. Biochem Biophys Res Commun 499:556-562 https://doi.org/10.1016/j.bbrc.2018.03.189

Mears JA, Lackner LL, Fang S, Ingerman E, Nunnari J, Hinshaw JE (2011) Conformational changes in Dnm1 support a contractile mechanism for mitochondrial fission. Nat Struct Mol Biol 18:20-26. https://doi.org/10.1038/nsmb.1949

Menne TF et al (2007) The Shwachman-Bodian-Diamond syndrome protein mediates translational activation of ribosomes in yeast. Nat Genet 39:486-495. https://doi.org/10.1038/ng 1994

Mozdy AD, McCaffery JM, Shaw JM (2000) Dnm1p GTPasemediated mitochondrial fission is a multi-step process requiring the novel integral membrane component Fis1p. J Cell Biol $151: 367-380$

Neubauer M, Zhu Z, Penka M, Helmschrott C, Wagener N, Wagener J (2015) Mitochondrial dynamics in the pathogenic mold Aspergillus fumigatus: therapeutic and evolutionary implications. Mol Microbiol 98:930-945. https://doi.org/10.1111/mmi.13167

Numadate A, Mita Y, Matsumoto Y, Fujii S, Hashimoto Y (2014) Development of 2-thioxoquinazoline-4-one derivatives as dual and selective inhibitors of dynamin-related protein 1 (Drp1) and puromycin-sensitive aminopeptidase (PSA). Chem Pharm Bull 62:979-988

Nunnari J, Suomalainen A (2012) Mitochondria: in sickness and in health. Cell 148:1145-1159. https://doi.org/10.1016/j. cell.2012.02.035

Ong SB, Subrayan S, Lim SY, Yellon DM, Davidson SM, Hausenloy DJ (2010) Inhibiting mitochondrial fission protects the heart against ischemia/reperfusion injury. Circulation 121:2012-2022. https://doi.org/10.1161/circulationaha.109.906610

Otsuga D, Keegan BR, Brisch E, Thatcher JW, Hermann GJ, Bleazard W, Shaw JM (1998) The dynamin-related GTPase, Dnm1p, controls mitochondrial morphology in yeast. J Cell Biol 143:333-349

Prevost CT, Peris N, Seger C, Pedeville DR, Wershing K, Sia EA, Sia RAL (2018) The influence of mitochondrial dynamics on mitochondrial genome stability. Curr Genet 64:199-214. https://doi. org/10.1007/s00294-017-0717-4

Qi X, Qvit N, Su YC, Mochly-Rosen D (2013) A novel Drp1 inhibitor diminishes aberrant mitochondrial fission and neurotoxicity. J Cell Sci 126:789-802. https://doi.org/10.1242/jcs.114439

Qian W, Ma D, Xiao C, Wang Z, Zhang J (2012) The genomic landscape and evolutionary resolution of antagonistic pleiotropy in yeast. Cell Rep 2:1399-1410. https://doi.org/10.1016/j.celre p.2012.09.017

Rambold AS, Kostelecky B, Elia N, Lippincott-Schwartz J (2011) Tubular network formation protects mitochondria from autophagosomal degradation during nutrient starvation. Proc Natl Acad Sci USA 108:10190-10195. https://doi.org/10.1073/ pnas. 1107402108

Rappold PM et al (2014) Drp1 inhibition attenuates neurotoxicity and dopamine release deficits in vivo. Nat Commun 5:5244. https:// doi.org/10.1038/ncomms6244

Rehman J et al (2012) Inhibition of mitochondrial fission prevents cell cycle progression in lung cancer. FASEB 26:2175-2186. https:// doi.org/10.1096/fj.11-196543

Rosdah AA, J KH, Delbridge LM, Dusting GJ, Lim SY (2016) Mitochondrial fission - a drug target for cytoprotection or cytodestruction? Pharmacol Res Perspect 4:e00235. https://doi.org/10.1002/ prp2.235

Ruf D, Brantl V, Wagener J (2018) Mitochondrial fragmentation in Aspergillus fumigatus as early marker of granulocyte killing activity. Front Cell Infect Microbiol 8:128 https://doi.org/10.3389/ fcimb.2018.00128 
Santos MMS, Elsztein C, De Souza RB, Paiva SSL Jr, Silva JA, Crovella S, De Morais MA Jr (2018) Respiratory deficiency in yeast mevalonate kinase deficient may explain MKD-associate metabolic disorder in humans. Curr Genet 64:871-881. https://doi. org/10.1007/s00294-018-0803-2

Sharp WW, Beiser DG, Fang YH, Han M, Piao L, Varughese J, Archer SL (2015) Inhibition of the mitochondrial fission protein dynamin-related protein 1 improves survival in a murine cardiac arrest model. Crit Care Med 43:e38-e47. https://doi.org/10.1097/ ccm.0000000000000817

Smith G, Gallo G (2017) To mdivi-1 or not to mdivi-1: is that the question? Dev Neurobiol 77:1260-1268. https://doi.org/10.1002/ dneu.22519

Song W et al (2011) Mutant huntingtin binds the mitochondrial fission GTPase dynamin-related protein-1 and increases its enzymatic activity. Nat Med 17:377-382. https://doi.org/10.1038/nm.2313

Sudbery PE (2011) Growth of Candida albicans hyphae. Nat Rev Microbiol 9:737-748. https://doi.org/10.1038/nrmicro2636

Sun S et al (2016) An extended set of yeast-based functional assays accurately identifies human disease mutations. Genome Res 26:670-680. https://doi.org/10.1101/gr.192526.115

Tieu Q, Nunnari J (2000) Mdv1p is a WD repeat protein that interacts with the dynamin-related GTPase, Dnm1p, to trigger mitochondrial division. J Cell Biol 151:353-366

Tieu Q, Okreglak V, Naylor K, Nunnari J (2002) The WD repeat protein, Mdv1p, functions as a molecular adaptor by interacting with Dnm1p and Fis1p during mitochondrial fission. J Cell Biol 158:445-452. https://doi.org/10.1083/jcb.200205031

Tucey TM et al (2018) Glucose homeostasis is important for immune cell viability during candida challenge and host survival of systemic fungal. Infect Cell Metabol 27:988-1006.e1007. https://doi. org/10.1016/j.cmet.2018.03.019 van Pel DM et al (2013) An evolutionarily conserved synthetic lethal interaction network identifies FEN1 as a broad-spectrum target for anticancer therapeutic development. PLoS Genet 9:e1003254. https://doi.org/10.1371/journal.pgen.1003254

Voelz K, Johnston SA, Smith LM, Hall RA, Idnurm A, May RC (2014) 'Division of labour' in response to host oxidative burst drives a fatal Cryptococcus gattii outbreak. Nat Commun 5:5194 https:// doi.org/10.1038/ncomms6194

Wang X et al (2008) Amyloid-beta overproduction causes abnormal mitochondrial dynamics via differential modulation of mitochondrial fission/fusion proteins. Proc Natl Acad Sci USA 105:1931819323. https://doi.org/10.1073/pnas.0804871105

Wang W et al (2017) Inhibition of mitochondrial fragmentation protects against Alzheimer's disease in rodent model. Hum Mol Genet 26:4118-4131. https://doi.org/10.1093/hmg/ddx299

Xie Q et al (2015) Mitochondrial control by DRP1 in brain tumor initiating cells. Nat Neurosci 18:501-510. https://doi.org/10.1038/ nn.3960

Zhong K et al (2016) MoDnm1 dynamin mediating peroxisomal and mitochondrial fission in complex with MoFis 1 and MoMdv1 is important for development of functional appressorium in Magnaporthe oryzae. PLoS Pathog 12:e1005823 https://doi.org/10.1371/ journal.ppat. 1005823

Publisher's Note Springer Nature remains neutral with regard to jurisdictional claims in published maps and institutional affiliations. 